Laser Chem. 1988, Vol. 8, pp. 97-122

(C) 1988 Harwood Academic Publishers GmbH

Photocopying permitted by license only

Reprints available directly from the Publisher

Printed in the United Kingdom

\title{
Raman spectroscopy of infrared multiphoton excited molecules
}

\author{
Jyhpyng Wang, Kuei-Hsien Chen and Eric Mazur \\ Division of Applied Sciences and Department of Physics \\ Harvard University, Cambridge, MA 02138, USA
}

(Received August 14, 1987; in final form September 15, 1987)

This paper presents an overview of data obtained on the intramolecular vibrational energy distribution in infrared multiphoton excited $\mathrm{CF}_{2} \mathrm{HCl}, \mathrm{CF}_{2} \mathrm{Cl}_{2}, \mathrm{SF}_{6}$ and $\mathrm{CH}_{3} \mathrm{CHF}_{2}$. All but $\mathrm{CF}_{2} \mathrm{HCl}$ show collisionless changes in the intensity of the spontaneous Raman signals after excitation, indicating that the excitation alters the population in the Raman active modes. A comparison of the spectrally integrated intensities of the Raman signals yields information on the distribution of vibrational energy over the modes of the molecule. The results for $\mathrm{CF}_{2} \mathrm{Cl}_{2}$ show a nonthermal distribution of energy after the excitation.

KEY WORDS: Infrared; multiphoton; $\mathrm{CF}_{2} \mathrm{HCl} ; \mathrm{CF}_{2} \mathrm{Cl}_{2} ; \mathrm{SF}_{6} ; \mathrm{CH}_{3} \mathrm{CHF}_{2} ;$ Raman spectroscopy.

\section{Introduction}

Most polyatomic molecules with a strong vibrational absorption band can absorb many (10 to 40) infrared photons when irradiated with an intense resonant infrared laser pulse. At high excitation many dissociate without interacting with other molecules. The early work in this field was motivated by the hope of driving chemical reactions in either a bond-specific or isotopically selective fashion. ${ }^{1}$ This could be achieved if some of the initial energy deposition were 'localized' in a small subset of modes. In the past decade much work ${ }^{2-7}$ has been directed toward gaining a better understanding of the infrared multiphoton excitation and dissociation of polyatomic molecules and the intramolecular dynamics of highly vibrationally excited molecules in general.

Many experimental techniques have been applied to study infrared multiphoton excitation. Photoacoustic measurements were applied to 


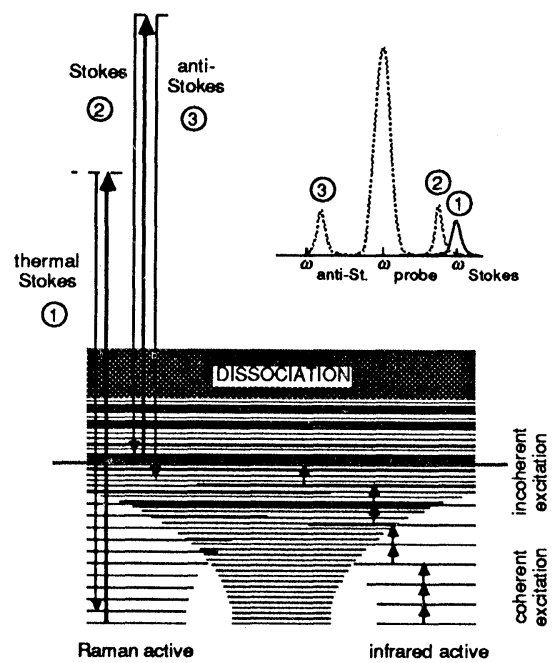

Figure 1 Spontaneous Raman spectroscopy of infrared multiphoton excited molecules. Molecules that remain in the lower vibrational states (cold molecules) show only Stokes scattering (1), while the highly excited ones (hot molecules) show both a shifted Stokes (2) and an anti-Stokes signal (3).

determine the energy absorbed by the molecules, ${ }^{8}$ and to study the excitation as a function of various parameters, such as pumping fluence, intensity, and wavelength, pressure, etc. Photoacoustic spectroscopy was also used at high intensities to study dissociation yields as a function of absorbed energy. More detailed information on infrared multiphoton dissociation, such as the species of the dissociation fragments, branching ratios of different dissociation channels, and the translational energy distribution of the fragments, was obtained by mass and time-of-flight spectrometry. ${ }^{9}$ Pump-and-probe experiments have also provided more detailed knowledge of the infrared multiphoton excitation and dissociation. For example, laser induced fluorescence ${ }^{10,11}$ was used to measure the vibrational energy distribution of the infrared multiphoton dissociation fragments. Infrared doubleresonance experiments ${ }^{12-14}$ were done to determine the rotational relaxation rate and the population depletion of the vibrational ground state. Spontaneous and coherent anti-Stokes Raman scattering were used to probe the distribution of vibrational energy over the different modes of infrared multiphoton excited molecules. ${ }^{15-21}$ 
In this paper we report on results obtained by time-resolved spontaneous Raman spectroscopy. The principle of the experiments, which were pioneered in the Soviet Union, ${ }^{15-20}$ is illustrated in Fig. 1. First, the molecules are pumped into the high vibrational excitation region by a short, intense $\mathrm{CO}_{2}$ laser pulse resonant with a vibrational mode. Then the Raman signals from the different Raman active modes accessible to the apparatus are measured with a second, ultraviolet laser pulse. While unexcited molecules show only a Stokes signal (Fig. 1, 1), highly excited ones show both a shifted Stokes signal (Fig. 1, 2) as well as an anti-Stokes (Fig. 1, 3) signal. Since the total, spectrally integrated signal from a Raman active mode is a measure of the amount of energy in the mode (see discussion), the intramolecular vibrational energy distribution after the excitation can be determined by comparing the Raman signals from different modes. Timeresolved information is obtained by varying the time delay between pump and probe.

\section{Experimental}

The experimental procedure is described in detail in previous publications. ${ }^{22,23}$ Briefly, molecules contained within a low pressure gas cell are excited by an infrared laser pulse and probed by an ultraviolet laser pulse. To isolate intramolecular from (collisional) intermolecular effects, the signals are measured at pressures low enough to ensure that no significant collisional relaxation of vibrational energy occurs on the time scale of the experiment.

A schematic view of the setup is shown in Fig. 2. The infrared radiation is generated by a high power short-pulse tunable $\mathrm{CO}_{2}$ laser with 0.5 to $15 \mathrm{~ns}$ pulse duration and a maximum energy of $200 \mathrm{~mJ}$. A $20 \mathrm{~ns}$ frequency-doubled ruby laser pulse of $3 \mathrm{~mJ}$ serves as Raman probe. The infrared exciting beam and an ultraviolet probing beam are focused inside the scattering cell 23 where they cross at right angles. The spontaneous Raman signals from the interaction region are detected perpendicular to the incident beams with a double monochromator and a high gain fast photomultiplier tube. The spectral resolution is $1.5 \mathrm{~nm}$, which is high enough to resolve the different Raman active modes, and low enough to integrate the signals over the states within one mode. A complete description of the experimental setup can be found in previous papers. 22,23

Time resolution is obtained by varying the time delay between pump and probe. The synchronization of lasers, which is controlled electronically, is 


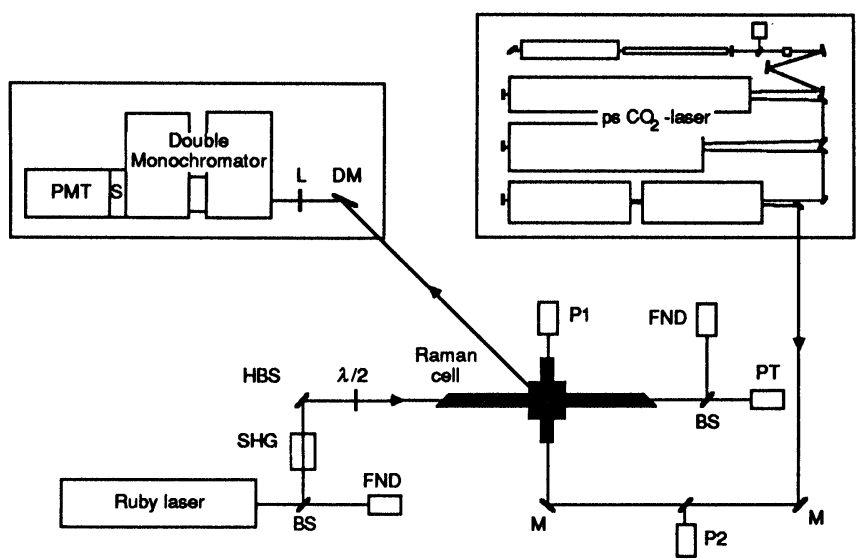

Figure 2 Experimental setup. BS = beam splitter, $\mathrm{SHG}=$ second harmonic generator, $\mathrm{HBS}=$ harmonic beam splitter, $\lambda / 2=$ half-wave plate, $\mathrm{FND}=$ fast photodiode, $\mathrm{PT}=$ phototube, $\mathrm{P} 1, \mathrm{P} 2=$ pyroelectric detector, $\mathrm{M}=$ mirror, $\mathrm{DM}=$ dichroic mirror, $\mathrm{L}=$ quartz lens, $\mathrm{S}=$ shutter, $\mathrm{PMT}=$ photomultiplier tube.

limited by the $100 \mathrm{~ns}$ trigger jitter of the lasers. To obtain time-resolved data on a shorter time scale, the actual time delay between infrared and ultraviolet pulses is measured for each pair of laser pulses with two fast detectors and a time-to-pulse-height converter. The time resolution of the setup is thus determined by the duration of the laser pulses.

At high pumping intensity a fraction of the molecules dissociates, and the probe laser can induce a fluorescence from the dissociation fragments. This laser induced fluorescence is much more intense than the spontaneous Raman scattering. One can discriminate between fluorescence and Raman scattering either spectrally or temporally. The fluorescence generally has a broad continuous spectrum and a long decay, while the spectrally discrete Raman signals coincide with the $20 \mathrm{~ns}$ probe pulse. Since the present measurements are carried out with the monochromator at fixed wavelengths, only temporal discrimination can be applied. The coincidence of the Raman signals with the probe pulse is therefore monitored throughout the experiment to ensure that no fluorescence contributes to the observed signals. In addition, the infrared and ultraviolet laser energy, the time delay between pump and probe, and the signal intensity are recorded for each laser pulse. 
Owing to the small Raman cross sections and the low sample pressure required to satisfy collisionless conditions, the signals are in the single photon regime. Consequently, a large amount of data has to be collected and averaged to obtain a satisfactory signal-to-noise ratio. A microcomputer collects the data, sorts out the data points according to infrared pump intensity and time delay between pump and probe and averages the data.

\section{Results}

Experiments were done at room temperature on $\mathrm{SF}_{6}, \mathrm{CF}_{2} \mathrm{HCl}, \mathrm{CF}_{2} \mathrm{Cl}_{2}$ and $\mathrm{CH}_{3} \mathrm{CHF}_{2}$ at pressures ranging from $33 \mathrm{~Pa}$ to $400 \mathrm{~Pa}$. All gases were obtained commercially and have a reported purity better than $99.99 \%$. Relevant spectroscopic data are given in Table I.

$\mathrm{CF}_{2} \mathrm{Cl}_{2}$. The infrared active $v_{8}$-mode of $\mathrm{CF}_{2} \mathrm{Cl}_{2}$, which has been assigned to the $\mathrm{CCl}_{2}$ asymmetric stretch, was pumped with $15 \mathrm{~ns}$ full-width at halfmaximum pulses from the $\mathrm{P}(32)$ line of the $10.6 \mu \mathrm{m} \mathrm{CO} 2$ laser branch. The $15 \mathrm{~ns}$ truncated pulses have a $10-90 \%$ rise time of $10 \mathrm{~ns}$ and a short subnanosecond fall time. Raman signals were obtained for the $1098 \mathrm{~cm}^{-1} \mathrm{CF}_{2}$ symmetric stretch $v_{1}$-mode $\left(A_{1}\right)$, the $923 \mathrm{~cm}^{-1} \mathrm{CCl}_{2}$ asymmetric stretch $v_{8}$ mode $\left(B_{1}\right)$, and the $667 \mathrm{~cm}^{-1} \mathrm{CCl}_{2}$ symmetric stretch $\mathrm{v}_{2}$-mode $\left(A_{1}\right)$. The signals from all these modes change after infrared multiphoton excitation, allowing one to compare the intensity ratio with the thermal one. Another interesting feature of this molecule is that the $v_{8}$ pump mode is both infrared and Raman active, allowing a direct view of the excitation in the pump mode.

$S F_{6}$. Results on this molecule, which are presented here for comparison, were published previously. ${ }^{22}$ The data were obtained at $\mathrm{CO}_{2}$ laser frequencies between the $\mathrm{P}(12)$ and $\mathrm{P}(28)$ lines of the $10.6 \mu \mathrm{m} \mathrm{CO} 2$ laser branch, which are resonant with the triply degenerate infrared active $v_{3}$-mode $\left(F_{1 u}\right)$ of $\mathrm{SF}_{6}$. Two different pulse durations were employed: short $0.5 \mathrm{~ns}$ and truncated $15 \mathrm{~ns}$ pulses with fluences up to $7 \times 10^{4} \mathrm{~J} / \mathrm{m}^{2}$. The Raman signals were obtained at a shift of $775 \mathrm{~cm}^{-1}$ from the frequency-doubled ruby laser, corresponding to the totally symmetric breathing mode $v_{1}$-mode $\left(A_{1 g}\right)$ of $\mathrm{SF}_{6}$.

$\mathrm{CF}_{2} \mathrm{HCl}$. The peak absorption of this molecule coincides with the $9.4 \mu \mathrm{m}$ $\mathrm{R}(32) \mathrm{CO}_{2}$ laser line at $1086 \mathrm{~cm}^{-1}$. Although five Raman active modes of $\mathrm{CF}_{2} \mathrm{HCl}$ are accessible to our apparatus, none of them shows any change 
Table I. Spectroscopic data for the molecules studied in this paper. The vibrational data for $\mathrm{SF}_{6}$ and $\mathrm{CF}_{2} \mathrm{Cl}_{2}$ are from literature. All data are in $\mathrm{cm}^{-1}$, vs = very strong, $\mathrm{s}=$ strong, $\mathrm{m}=$ medium, and $\mathrm{w}=$ weak.

\begin{tabular}{|c|c|c|c|c|c|}
\hline Molecule & $\mathrm{CO}_{2}$ line & Wavenumber & Mode & Activity & Remarks \\
\hline \multirow[t]{6}{*}{$\mathrm{SF}_{6} 25$} & & & $v_{1}=775$ & $\mathrm{R}(\mathrm{s})$ & changes \\
\hline & & & $v_{2}=644$ & $R(w)$ & not probed \\
\hline & $10.6 \mu \mathrm{m} \mathrm{P}(20)$ & 944 & $v_{3}=965$ & IR & pumped \\
\hline & & & $v_{4}=617$ & IR & \\
\hline & & & $v_{5}=524$ & $R(w)$ & not probed \\
\hline & & & $v_{6}=363$ & inactive & \\
\hline \multirow[t]{14}{*}{$\mathrm{CF}_{2} \mathrm{Cl}_{2}{ }^{26}$} & 6,27 & & $v_{1}=1101$ & $\operatorname{IR}(\mathrm{s})$ & \\
\hline & & & $v_{1}=1098$ & $R(m)$ & changes \\
\hline & & & $v_{2}=667$ & $\mathrm{IR}(\mathbf{s})$ & \\
\hline & & & $v_{2}=667.2$ & $R(s)$ & changes \\
\hline & & & $v_{3}=457.5$ & $\mathbf{R}(\mathrm{s})$ & not probed \\
\hline & & & $v_{4}=261.5$ & $\mathrm{R}(\mathrm{s})$ & not probed \\
\hline & & & $v_{5}=322$ & $R(w)$ & not probed \\
\hline & & & $v_{6}=1159$ & $\mathbb{R}(s)$ & \\
\hline & & & $v_{6}=1167$ & $R(w)$ & not probed \\
\hline & & & $v=446$ & $\operatorname{IR}(w)$ & not probed \\
\hline & $10.6 \mu \mathrm{m} \mathrm{P}(32)$ & 933 & $v_{8}=922$ & IR (vs) & pumped \\
\hline & & & $v_{8}=923$ & $\mathbf{R}(w)$ & changes \\
\hline & & & $v_{9}=437$ & IR (w) & \\
\hline & & & $v g=433$ & $R(m)$ & not probed \\
\hline \multirow[t]{5}{*}{$\mathrm{CH}_{3} \mathrm{CHF}_{2}$} & & & 870 & $\mathbf{R}$ & changes \\
\hline & $10.6 \mu \mathrm{m} \mathrm{P}(20)$ & 944 & & IR & pumped \\
\hline & & & 1140 & $\mathbf{R}$ & no change \\
\hline & & & 1460 & $\mathbf{R}$ & no change \\
\hline & & & 2980 & $\mathbf{R}$ & no change \\
\hline \multirow[t]{6}{*}{$\mathrm{CF}_{2} \mathrm{HCl}$} & & & 590 & $\mathbf{R}$ & no change \\
\hline & & & 800 & $\mathbf{R}$ & no change \\
\hline & $9.4 \mu \mathrm{m} \mathrm{R}(32)$ & 1086 & & IR & pumped \\
\hline & & & 1130 & $\mathbf{R}$ & no change \\
\hline & & & 1330 & $\mathbf{R}$ & no change \\
\hline & & & 3030 & $\mathbf{R}$ & no change \\
\hline
\end{tabular}


after infrared multiphoton excitation with $0.5 \mathrm{~ns}$ pulses, even at fluences up to $2 \times 10^{4} \mathrm{~J} / \mathrm{m}^{2}$, when the average number of photons absorbed per molecule is reported to be about ten. ${ }^{24}$

$\mathrm{CH}_{3} \mathrm{CHF}_{2}$. The largest of the molecules studied, $\mathrm{CH}_{3} \mathrm{CHF}_{2}$, has four accessible Raman active modes. Only one of these modes, at $870 \mathrm{~cm}^{-1}$, shows a small anti-Stokes signal after infrared multiphoton excitation. The molecules were pumped with $0.5 \mathrm{~ns}$ pulses at the $\mathrm{P}(20)$ line of the $10.6 \mu \mathrm{m}$ branch.

Some of the experimental results are shown in Figs. 3 through 13 and listed in Tables I, II and III. The results are arranged so as to emphasize the similarities and the differences in behavior between the molecules. The points shown in each of the figures are obtained by dividing the $x$-axis into a number of intervals (usually 10 to 20), and averaging the data that lie within each of the intervals. Since the signal fluctuations in the single photon regime are large and since the error bars are inversely proportional to the square root of the number of points, the average is taken over a large number of data points. The low repetition rate of $0.3 \mathrm{~Hz}$ and the stability of the alignment, however, limit the total number of pulses for a single experimental run to $10^{4}$. This means that there is a trade-off between the number of points in each figure and the length of the error bars. In all measurements presented here the error bars are about $10 \%$ of the absolute value of the data points for $\mathrm{SF}_{6}$ and, because of the smaller Raman cross-sections, $20 \%$ for $\mathrm{CF}_{2} \mathrm{Cl}_{2}$ and $\mathrm{CH}_{3} \mathrm{CHF}_{2}$.

The Raman spectra of infrared multiphoton excited $\mathrm{SF}_{6}$ and $\mathrm{CF}_{2} \mathrm{Cl}_{2}$ at a pressure of $400 \mathrm{~Pa}$ are shown in Figs. 3 and 4. The large central peak in the spectrum corresponds to Rayleigh scattering at the probe laser wavelength. The single Raman peak at each side of the Rayleigh peak in Fig. 3 corresponds to the $v_{1}$-mode of $\mathrm{SF}_{6}$. At room temperature without infrared excitation only Stokes signals are observable (open circles). If the molecules are excited before the Raman probing (closed circles) anti-Stokes signals appear in the spectrum. Of the nine vibrational modes of $\mathrm{CF}_{2} \mathrm{Cl}_{2}$, three, at $667 \mathrm{~cm}^{-1}, 923 \mathrm{~cm}^{-1}$ and $1098 \mathrm{~cm}^{-1}$ respectively, are visible in the (low pressure, low resolution) spectrum in Fig. 4. Since the v3 mode at $923 \mathrm{~cm}^{-1}$ is both Raman and infrared active, $\mathrm{CF}_{2} \mathrm{Cl}_{2}$ allows direct monitoring of the pumped mode. Within the spectral resolution, the Raman shifts of the anti-Stokes signals that appear after excitation correspond to the ones reported in the literature. Note that for each of the detectable Raman peaks a corresponding anti-Stokes peak appears after excitation, both at an energy smaller than the excitation energy $\left(667 \mathrm{~cm}^{-1}\right)$ and also at higher en- 


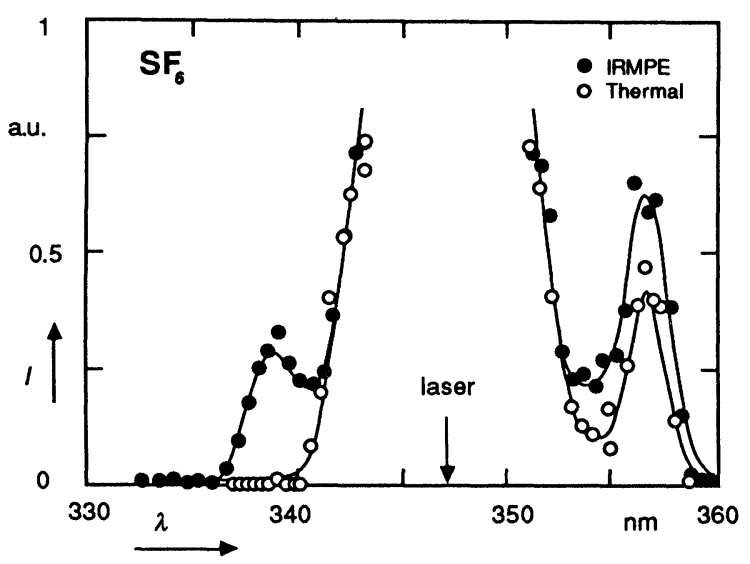

Figure 3 Raman spectrum of $\mathrm{SF}_{6}$, with (closed circles) and without (open circles) infrared multiphoton excitation. Infrared excitation: $10.6 \mu \mathrm{m} \mathrm{P}(20)$ line, $0.5 \mathrm{~ns}$ pulse duration, and average fluence $0.6 \times 10^{4} \mathrm{~J} / \mathrm{m}^{2}$. The small arrow marks the position of the probe laser radiation at $347.15 \mathrm{~nm}$.

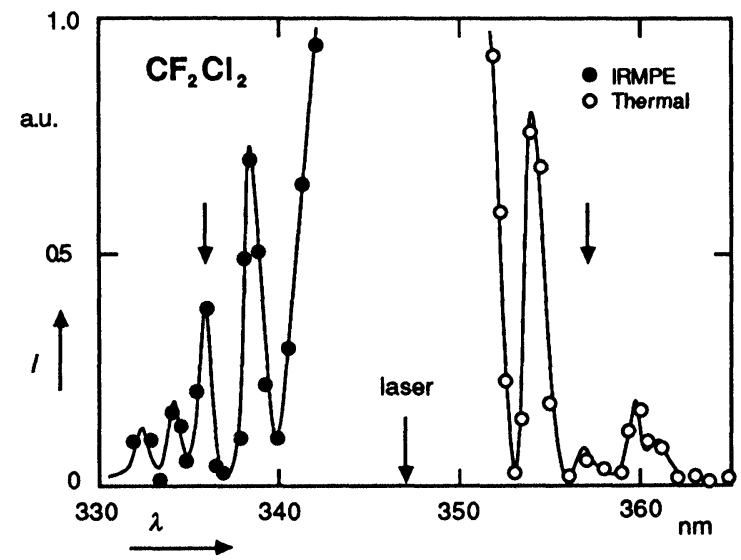

Figure 4 Raman spectrum of $\mathrm{CF}_{2} \mathrm{Cl}_{2}$, with (closed circles) and without (open circles) infrared multiphoton excitation. Infrared excitation with $15 \mathrm{~ns}$ pulses at the $10.6 \mu \mathrm{m}$ $P(32)$ line. The arrows mark the position of the probe laser and the infrared pump mode. 


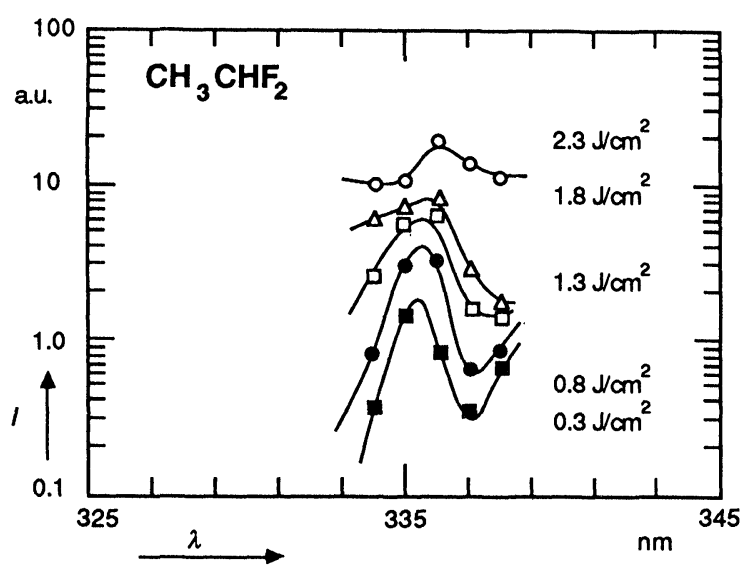

Figure 5 Anti-Stokes signal of the $870 \mathrm{~cm}^{-1}$ mode of infrared multiphoton excited $\mathrm{CH}_{3} \mathrm{CHF}_{2}$ at various fluences. Infrared excitation: $10.6 \mu \mathrm{m} \mathrm{P}(20)$ line, $0.5 \mathrm{~ns}$ pulse duration. At high fluence laser induced fluorescence from the dissociation fragments replaces the Raman signal.

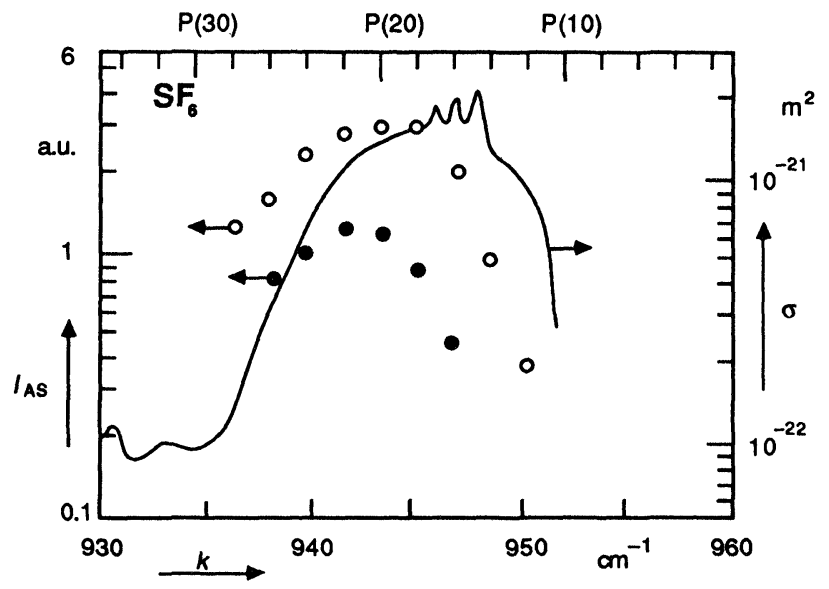

Figure 6 Anti-Stokes signal for $\mathrm{SF}_{6}$ from excitations at different $\mathrm{CO}_{2}$-lines. Data for two different pulse durations at an average fluence of $10^{4} \mathrm{~J} / \mathrm{m}^{2}$ are shown: $0.5 \mathrm{~ns}$ (open circles) and $15 \mathrm{~ns}$ (closed circles). The curve shows the one photon absorption cross section. 
ergy $\left(1098 \mathrm{~cm}^{-1}\right)$. At fluences above $3 \times 10^{4} \mathrm{~J} / \mathrm{m}^{2}$ a broadband laser induced fluorescence gradually replaces the Raman spectrum. This fluorescence is induced by the probe laser in the fragments of infrared multiphoton dissociated molecules.

Figure 5 shows the anti-Stokes signal of the $870 \mathrm{~cm}^{-1}$ mode of $\mathrm{CH}_{3} \mathrm{CHF}_{2}$ for different fluences. As the fluence is increased, the antiStokes peak is gradually replaced by a broadband laser induced fluorescence just as for $\mathrm{CF}_{2} \mathrm{Cl}_{2}$.

Figure 6 shows the anti-Stokes signal of $\mathrm{SF}_{6}$ for excitation at different $\mathrm{CO}_{2}$-lines. Each point shown represents a separate experimental run in which the anti-Stokes signal of infrared multiphoton excited $\mathrm{SF}_{6}$ was measured for a particular $\mathrm{CO}_{2}$-line. This graph shows the intensity of the anti-Stokes signal at a fluence of $10^{4} \mathrm{~J} / \mathrm{m}^{2}$ for each of the $\mathrm{CO}_{2}$-lines. As a reference the low signal absorption cross-section for $\mathrm{SF}_{6}$ is also plotted. Apart from a red shift of about $3 \mathrm{~cm}^{-1}$ from the center of the one photon absorption band, the absorption spectrum is not much different from the one at low excitation.

Figure 7 shows the time dependence of the Raman signal intensities for $\mathrm{SF}_{6}$ at $400 \mathrm{~Pa}$ for two infrared pulse durations, $0.5 \mathrm{~ns}$ and $15 \mathrm{~ns}$. The vertical axis shows the relative intensity, $I_{\text {rel }}$, obtained by normalizing the anti-Stokes signal after excitation with the room temperature equilibrium Stokes signal. The horizontal axis shows the time delay between pump and probe pulses. For negative time delay the molecules are probed before the infrared multiphoton excitation (i.e. at room temperature equilibrium) and only a Stokes signal, which serves as calibration for the intensity scale, is detectable. The rise time of the signals reflects the $30 \mathrm{~ns}$ instrumental time resolution, which in turn is determined by the temporal width of the laser pulses. Within the $30 \mathrm{~ns}$ time resolution a collisionless (see discussion) increase of both Stokes and anti-Stokes signals is observed. The signal remains constant up to $800 \mathrm{~ns}$ after the infrared excitation. The signals revert to their original values only after a much longer time $(10 \mu \mathrm{s})$ because of a combination of collisional relaxation and diffusion of the excited molecules out of the excitation region. ${ }^{22}$

A different behavior is observed for the other two molecules that exhibit changes in Raman spectrum. Figure 8 shows the time dependence of the three accessible Raman active modes of $\mathrm{CF}_{2} \mathrm{Cl}_{2}$ for infrared excitation with $15 \mathrm{~ns}$ pulses. Each of the anti-Stokes signals is normalized with its corresponding Stokes signal at room temperature. Just as for $\mathrm{SF}_{6}$, anti-Stokes signals appear for all three modes after excitation at $t=0$, but the signals decay much more rapidly, and although the time dependences are similar for the various modes, the maximum relative intensities differ. As can be seen 


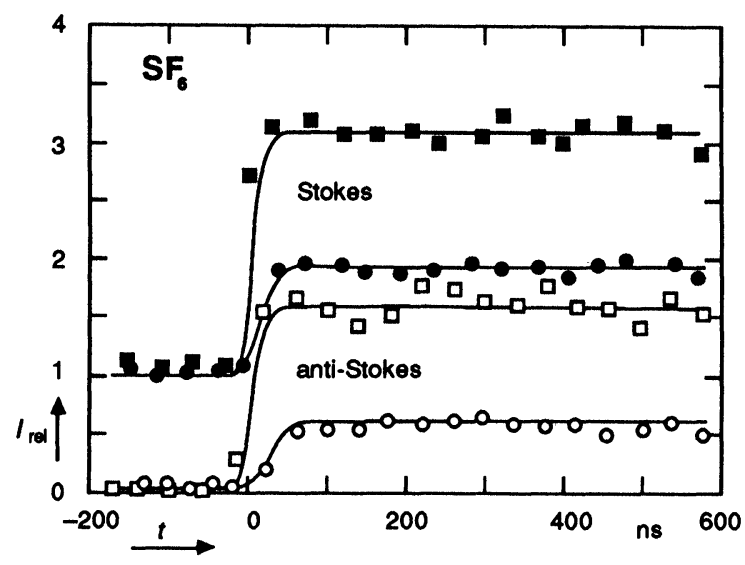

Figure 7 Intensity of Stokes (closed symbols) and anti-Stokes (open symbols) signal as a function of the time delay between pump and probe pulses at a pressure of $67 \mathrm{~Pa}$ for $\mathrm{SF}_{6}$. Infrared excitation with $0.5 \mathrm{~ns}$ (squares) and $15 \mathrm{~ns}$ (circles) pulses at the $10.6 \mu \mathrm{m}$ $\mathrm{P}(20)$ line. Average fluence: $0.8 \times 10^{4} \mathrm{~J} / \mathrm{m}^{2}$.

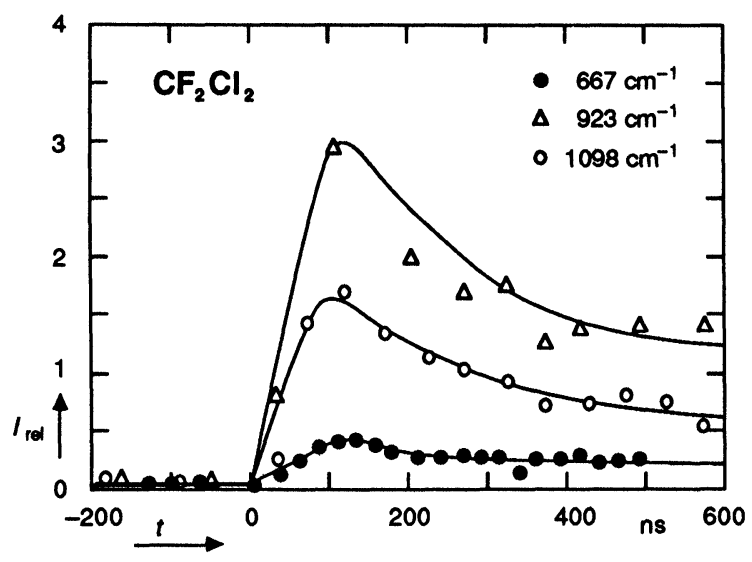

Figure 8 Intensity of the anti-Stokes signals as a function of the time delay between pump and probe pulse for $\mathrm{CF}_{2} \mathrm{Cl}_{2}$ at $400 \mathrm{~Pa}$. Infrared excitation: $10.6 \mu \mathrm{m} \mathrm{P}(32)$ line, $15 \mathrm{~ns}$ pulse with average fluence $1.8 \times 10^{4} \mathrm{~J} / \mathrm{m}^{2}$. 


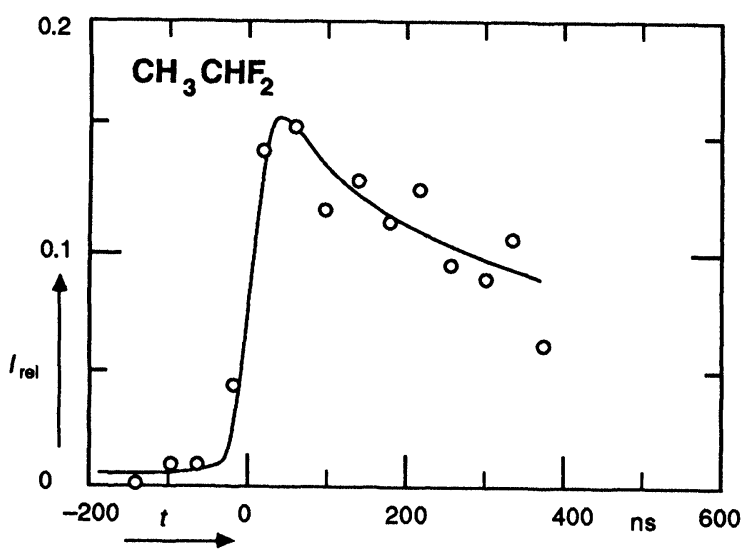

Figure 9 Intensity of the anti-Stokes signals as a function of the time delay between pump and probe pulse for $\mathrm{CH}_{3} \mathrm{CHF}_{2}$ at $660 \mathrm{~Pa}$. Infrared excitation: $10.6 \mu \mathrm{m} \mathrm{P}(20)$ line, $0.5 \mathrm{~ns}$ pulse with average fluence $1.5 \times 10^{4} \mathrm{~J} / \mathrm{m}^{2}$.

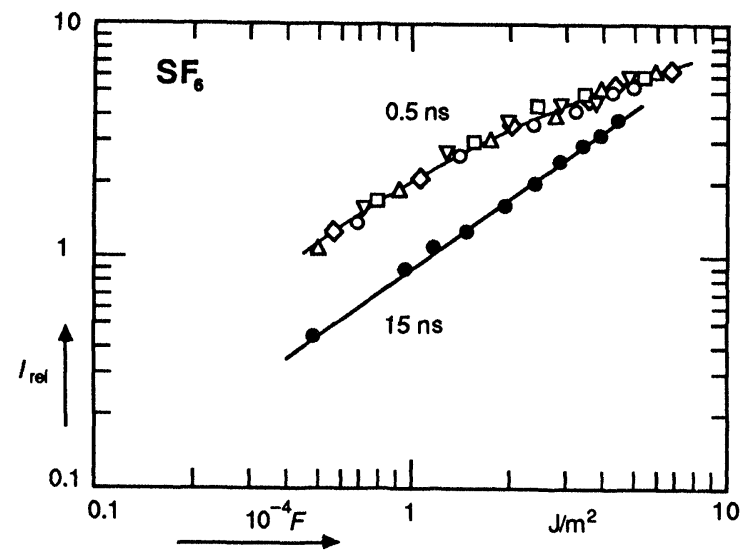

Figure 10 Relative anti-Stokes signal of $\mathrm{SF}_{6}$ as a function of the infrared pumping fluence for various pressures. Excitation at the $10.6 \mu \mathrm{m} \mathrm{P}(20)$ line with two pulse durations: $0.5 \mathrm{~ns}$ (open symbols) and $15 \mathrm{~ns}$ (closed symbols). ㅁ: $33 \mathrm{~Pa} ; \circ: 67 \mathrm{~Pa} ; \triangle: 133$ $\mathrm{Pa} ; \nabla: 200 \mathrm{~Pa} ; \diamond: 267 \mathrm{~Pa} ; \bullet: 133 \mathrm{~Pa}$. 
in Fig. 9, a similar behavior is observed for $\mathrm{CH}_{3} \mathrm{CHF}_{2}$. Note that for this molecule the change in $I_{\text {rel }}$ is ten times smaller than the one for the other two molecules.

The dependence of the normalized anti-Stokes signals on the infrared pumping fluence is shown in Figs, 10 and 11. The results for $\mathrm{SF}_{6}$, shown in Fig. 10, were obtained in different measurements, each one calibrated individually, at different pressures and pulse durations. The spread in data therefore shows the absolute accuracy and the reproducibility of the experimental data. As can be seen the dependence is nearly linear, except for some saturation effects at high fluence for the shorter (more intense) pulses. Also, the normalized anti-Stokes signals are independent of the sample gas pressure, as one would expect for collisionless effects.

Again, the results for $\mathrm{CF}_{2} \mathrm{Cl}_{2}$ and $\mathrm{CH}_{3} \mathrm{CHF}_{2}$ show a different behavior. Figure 11 shows the fluence dependence of the anti-Stokes signals for $\mathrm{CF}_{2} \mathrm{Cl}_{2}$. The data were obtained in a single measurement by changing the monochromator wavelength every two thousand laser shots (corresponds to about two hours in time) and averaging the data in the 0 to $500 \mathrm{~ns}$ range. In contrast to the linear fluence dependence of $\mathrm{SF}_{6}$, all three modes have an exponential fluence dependence. The graph also shows clearly that the rate of increase is drastically different for the three modes. A similar exponential fluence dependence was observed for $\mathrm{CH}_{3} \mathrm{CHF}_{2}$.

The normalized anti-Stokes signals of $\mathrm{CF}_{2} \mathrm{Cl}_{2}$ were also studied in the presence of $\mathrm{N}_{2}$ buffer gas. Figure 12 shows the intensity ratio of the antiStokes signals with and without buffer gas. Each of the anti-Stokes signals rapidly decreases with increasing buffer gas pressure, while at the same time the differences between them become smaller. No data are available for the anti-Stokes signal at $923 \mathrm{~cm}^{-1}$ at $26 \mathrm{kPa}$ buffer gas pressure. Since the Raman cross-section of the $923 \mathrm{~cm}^{-1}$ Raman transition is much smaller than that of the other two modes, this peak falls below the noise level at high buffer gas pressure.

Figure 13 shows the spectrum of the laser induced fluorescence from the infrared multiphoton dissociated $\mathrm{CF}_{2} \mathrm{Cl}_{2}$ at three different fluences ranging from 4 to $6 \times 10^{4} \mathrm{~J} / \mathrm{m}^{2}$. The fluorescence signal was recorded in $10 \mathrm{~nm}$ increments from $290 \mathrm{~nm}$ to $340 \mathrm{~nm}$. Although the intensity increases with increasing fluence, the spectral shape of the broadband emission does not change. In addition it should be noted that the fluorescence extends into the blue side of the incident laser field at $347.15 \mathrm{~nm}$. The cut-off wavelength of the fluorescence is about $290 \mathrm{~nm}$, which means that the dissociation fragments carry as much as $5000 \mathrm{~cm}^{-1}$, or about 5 infrared photons, of internal energy. 


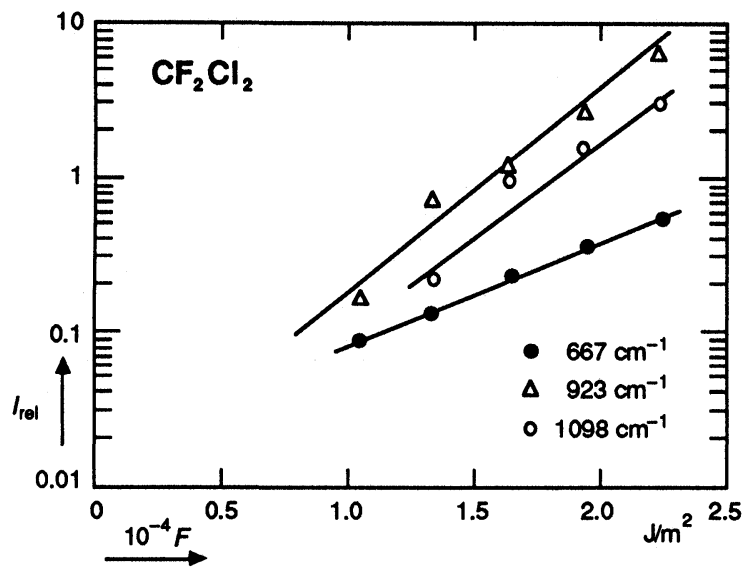

Figure 11 Semilogarithmic plot of the fluence dependence of the anti-Stokes signals of $\mathrm{CF}_{2} \mathrm{Cl}_{2}$ at $400 \mathrm{~Pa}$. The dependence is exponential for all three modes. Infrared excitation with $15 \mathrm{~ns}$ pulses at the $10.6 \mu \mathrm{m} \mathrm{P}(32)$ line.

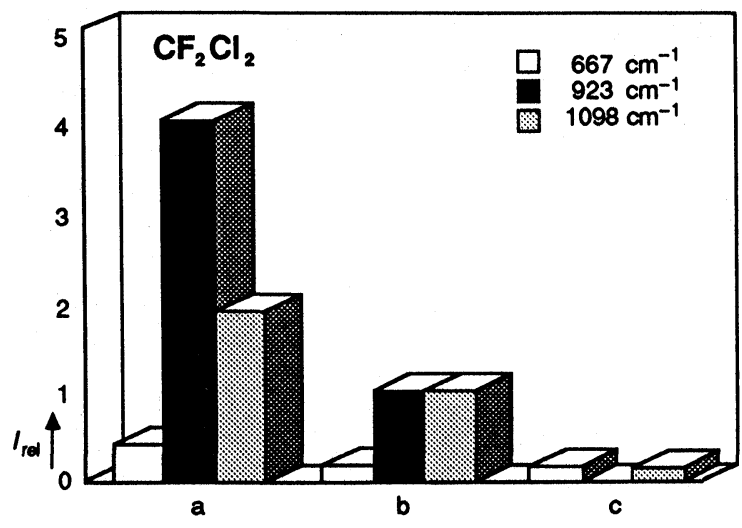

Figure 12 Comparison of anti-Stokes intensities for $\mathrm{CF}_{2} \mathrm{Cl}_{2}$ at an average fluence of 2.1 $\times 10^{4} \mathrm{~J} / \mathrm{m}^{2}$ and a pressure of $400 \mathrm{~Pa}$, without buffer gas $(a)$, and with $13 \mathrm{kPa}(b)$, and 26 $\mathrm{kPa}(c)$ of $\mathrm{N}_{2}$ buffer gas. 


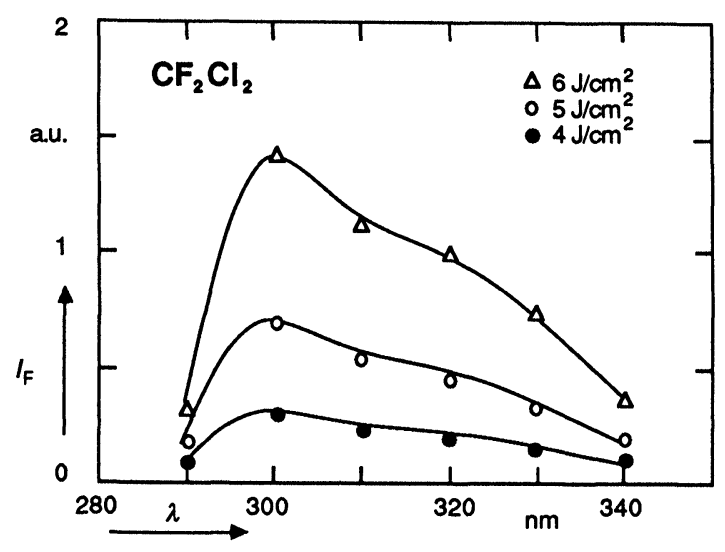

Figure 13 Laser induced fluorescence spectrum from the dissociation fragments of $\mathrm{CF}_{2} \mathrm{Cl}_{2}$. Note that only the intensity of the fluorescence increases with increasing fluence; the spectral shape does not change.

\section{Discussion}

The results in the previous sections show that infrared multiphoton excitation induces significant changes in the Raman signal intensities of the molecules. In this section we will analyze these changes and interpret the results in terms of a simple picture. This will allow us to obtain information on the distribution of energy among the various modes of the multiphoton excited molecules.

In the approximation that the vibrational mode is harmonic it can readily be shown that the transition probabilities $W$ of the Raman transitions depend on the vibrational quantum number $n$. For Stokes and anti-Stokes transitions, respectively, one has 22

$$
W_{n \rightarrow n+1} \sim n+1, \text { and } W_{n \rightarrow n-1} \sim n .
$$

The total intensity of the spontaneous Stokes and anti-Stokes Raman signals (summed over all vibrational levels of the vibrational mode considered), $I_{\mathrm{S}}$ and $I_{\mathrm{aS}}$ respectively, are given by 


$$
\begin{aligned}
& I_{\mathrm{S}} \sim \sum_{n=0}^{\infty} P(n) W_{n \rightarrow n+1} \sim 1+\sum_{n=0}^{\infty} P(n) n, \\
& I_{\mathrm{aS}} \sim \sum_{n=0}^{\infty} P(n) W_{n \rightarrow n-1} \sim \sum_{n=0}^{\infty} P(n) n,
\end{aligned}
$$

with $P(n)$ the population of level $n$. Substituting the average energy in the mode per molecule, $E_{\mathrm{R}}=h v_{\mathrm{R}} \sum P(n) n$, with $v_{\mathrm{R}}$ the frequency of the Raman active mode, one finds

$$
\begin{aligned}
& I_{\mathrm{S}} \sim 1+\frac{E_{\mathrm{R}}}{h v_{\mathrm{R}}}, \\
& I_{\mathrm{aS}} \sim \frac{E_{\mathrm{R}}}{h v_{\mathrm{R}}} .
\end{aligned}
$$

Note that the result obtained does not depend on the energy distribution $P(n)$, but only on the average energy $E_{\mathrm{R}}$.

The proportionality constant between the intensities $I_{S}$ and $I_{a S}$, and energy $E_{R}$, is related to the Raman scattering cross-section and is mode dependent. To eliminate this mode dependent quantity, the signals are normalized with the corresponding Stokes signal at room temperature, $I_{S}{ }^{\circ}$,

$$
I_{\mathrm{rel}} \equiv \frac{I_{\mathrm{aS}}}{I_{\mathrm{S}}^{\mathrm{o}}}=b^{-1} \sum_{n=\mathrm{o}}^{\infty} n P(n)=b^{-1} \frac{E_{\mathrm{R}}}{h v_{\mathrm{R}}},
$$

with $b \equiv \sum_{n=0}^{\infty}(n+1) P_{\mathrm{o}}(n)$,

where $P_{0}(n)$ is the population distribution at room temperature. If at room temperature $h v_{\mathrm{R}} \gg k T$, then the Boltzmann factor $e^{-h v / k T}$ is small, and $b \approx 1$. Under these circumstances the relative intensity of each mode is a direct measure of the average number of vibrational quanta, $\langle n\rangle=E_{\mathrm{R}} / h v_{\mathrm{R}}$, in that mode. The intensity ratio of the different Raman signals will therefore reflect the distribution of energy among the modes.

Questions have been raised about the influence of possible Fermi resonances on the interpretation of the Raman spectra of highly vibrationally 

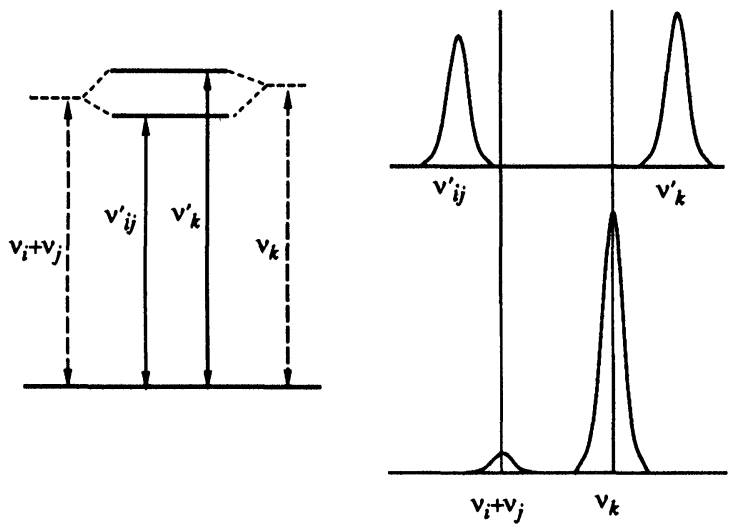

Figure 14 The effect of a Fermi resonance on the molecular energy levels and spectrum. The interaction between the $v_{i}+v_{j}$ combination and the $v_{k}$ fundamental transitions shifts the energy levels and redistributes the spectral intensity. The bottom spectrum is the expected one without Fermi resonance, the top one shows the actual spectrum.

excited molecules. ${ }^{28}$ The interaction of a fundamental with an overtone or with a combination mode may cause Fermi resonances when certain symmetry requirements are satisfied. ${ }^{29}$ Basically, if the frequency of an overtone or a combination mode happens to lie close to the frequency of a fundamental mode, and if the interaction between the two is strong enough, the nature of the two processes mix and the energy levels are displaced. As a result the vibrational spectrum can be misinterpreted. One well known example is the mixing of the $1300 \mathrm{~cm}^{-1}$ vibrational mode and the overtone of the $667 \mathrm{~cm}^{-1}$ vibrational mode of $\mathrm{CO}_{2}$, which causes two peaks, instead of one single peak, to appear in the Raman spectrum of $\mathrm{CO}_{2}$ around $1300 \mathrm{~cm}^{-1}$. Since this problem is related to the interpretation of the antiStokes signals of highly excited molecules in our experiment, the possible effect of Fermi resonances on our experimental data must be examined.

Let us consider an anti-Stokes transition in the $k$ th vibrational mode of a certain molecule. For simplicity we assume that the initial state $\psi_{k}$ corresponds to the first excited state of the $k$ th vibrational mode, and that the final state of the Raman transition is the ground state $\psi_{0}$. We further assume that the frequency $v_{k}$ of this Raman active vibrational mode lies close to the sum of the frequencies of two other fundamental modes, $v_{k} \approx v_{i}+v_{j}$, where $i, j$ represent the $i$ th and $j$ th vibrational modes respectively (see Fig. 14). In 
addition we assume that the interaction matrix element between the doubly excited combination state $\psi_{i j}$ and $\psi_{k}$

$$
W_{i j, k}=\int \psi_{i j}^{*} W \psi_{k} \mathrm{~d}^{3} x
$$

with $W$ the interaction operator, is nonzero. The actual energy levels $E^{\prime}=h v^{\prime}$, with $v^{\prime}=v_{i j}^{\prime}, v^{\prime}{ }_{k}$, of these interacting states will then be determined by the secular equation

$$
\left|\begin{array}{cc}
\left(v_{i}+v_{j}\right)-v^{\prime} & W_{i j, k} \\
W_{i j, k} & v_{k}-v^{\prime}
\end{array}\right|=0 .
$$

The wavefunctions of the interacting states will be superpositions of the original wavefunctions,

$$
\begin{aligned}
& \psi_{i j}^{\prime}=a \psi_{k}+b \psi_{i j} \\
& \psi_{k}^{\prime}=c \psi_{k}+d \psi_{i j},
\end{aligned}
$$

where the transformation matrix with coefficients $a, b, c$ and $d$ is unitary. These two equations show the effect of Fermi resonances on the intensities of the two transitions: the peak corresponding to the inherently weak combination mode $\psi_{i j}$ grows considerably because it 'borrows' intensity from the wavefunction of the fundamental mode $\psi_{k}$, while at the same time the intensity of the fundamental is reduced. Clearly, in high resolution spectroscopy the displacement of the line positions and the redistribution of line intensities between interacting combination and fundamental modes can be misleading.

In the present experiment, however, the situation is quite different. To accommodate the change in level spacing due to the anharmonicity of the vibrational mode, the measurements are done at low spectral resolution. Thus all Raman photons scattered from different excitation levels will be collected and integrated. If the displacements due to Fermi resonances are smaller than the resolution of the monochromator $(1.5 \mathrm{~nm})$ this means that one measures the spectrally integrated signal,

$$
\left.\left.\left\langle\psi_{i j}^{\prime}\left|\mathbb{R}_{i j}+\mathbb{R}_{k}\right| \psi_{0}\right\rangle\right|^{2}+K \psi^{\prime}{ }_{k}\left|\mathbb{R}_{i j}+\mathbb{R}_{k}\right| \psi_{0}\right\rangle\left.\right|^{2}=
$$




$$
\begin{aligned}
& \left|a^{*}\left\langle\psi_{k}\left|\mathscr{R}_{k}\right| \psi_{0}\right\rangle+b^{*}\left\langle\psi_{i j}\left|\mathscr{R}_{i j}\right| \psi_{0}\right\rangle\right|^{2}+\left|c^{*}\left\langle\psi_{k}\left|\mathscr{R}_{k}\right| \psi_{0}\right\rangle+d^{*}\left\langle\psi_{i j}\left|\mathscr{R}_{i j}\right| \psi_{0}\right\rangle\right|^{2}= \\
& \left|\left\langle\psi_{k}\left|\mathscr{R}_{k}\right| \psi_{0}\right\rangle\right|^{2}+\left|\left\langle\psi_{i j}\left|\mathscr{R}_{i j}\right| \psi_{0}\right\rangle\right|^{2} \approx\left|\left\langle\psi_{k}\left|\mathscr{R}_{k}\right| \psi_{0}\right\rangle\right|^{2}
\end{aligned}
$$

with $\mathcal{R}_{i j}$ and $\mathscr{R}_{k}$ the combination mode and Raman scattering operators, respectively, and where we have used the unitary properties of the transformation matrix. Equation(11) demonstrates that the spectrally integrated signal is identical to the one without Fermi resonance. Therefore the measured signal is the sum of the 'real' Raman signal $\left.K \psi_{k}\left|\mathbb{R}_{k}\right| \psi_{0}\right\rangle\left.\right|^{2}$ plus a much smaller quantity $\left.\left\langle\psi_{i j}\left|R_{i j}\right| \psi_{0}\right\rangle\right|^{2}$, whether or not Fermi resonances occur.

In addition to this general observation, a closer look at the available spectroscopic data for $\mathrm{SF}_{6}$ and $\mathrm{CF}_{2} \mathrm{Cl}_{2}$ further reveals that there are no Fermi resonances with the Raman modes studied here. The only candidate for a Fermi resonance with the $775 \mathrm{~cm}^{-1}$ mode of $\mathrm{SF}_{6}$ is the overtone of the $363 \mathrm{~cm}^{-1}$ vibrational mode. However the $363 \mathrm{~cm}^{-1}$ vibrational mode is spectroscopically inactive because of its $F_{2 u}$ symmetry. For $\mathrm{CF}_{2} \mathrm{Cl}_{2}$, the only reported Fermi resonance is due to the combination mode $v_{3}+v_{9}$ $\left(882 \mathrm{~cm}^{-1}\right)$, which is infrared, not Raman active.

Summarizing the above remarks, we may conclude that Fermi resonances cannot affect our experimental data. In what follows the observed anti-Stokes signals are therefore assumed to be correctly assigned and the intensity is used as a measure of the average number of vibrational quanta in each of the modes.

Time dependence. The signal increase in Fig. 7 is consistent with the result obtained in Eq. (3-4), i.e. for each pulse duration both Stokes and anti-Stokes signal increase by the same amount. Surprisingly, after the initial increase the signals remain constant, even on a time scale on which collisional vibrational energy relaxation is known to play a role. ${ }^{30}$ Clearly, collisions do not affect the total intensity of the anti-Stokes signal. Since the intensity of the signal is determined by the average energy in the mode only, intermolecular vibrational energy relaxation will not affect the Raman signals once intramolecular equilibrium is reached. This suggests that intramolecular equilibrium is reached on a time scale shorter than the time resolution.

The time dependence of the Raman signals for $\mathrm{CF}_{2} \mathrm{Cl}_{2}$ shown in Fig. 8 is quite different. The signals do not remain constant as for $\mathrm{SF}_{6}$, especially for the two highly excited modes $\left(923 \mathrm{~cm}^{-1}\right.$ and $\left.1098 \mathrm{~cm}^{-1}\right)$. This decay is most likely due to collisional transfer of energy to other, initially 'cold', 
vibrational modes. Because of the limited sensitivity and spectral range, however, this cannot be verified in the present experimental setup.

Pressure dependence. Although the increase in Raman signals takes place on a time scale small compared to the mean free time of the sample molecules, collisions cannot be completely eliminated. To verify the absence of collisional effects, the dependence of the Raman signal on sample gas pressure was measured. It was indeed found that the normalized Raman signals do not depend on pressure. 22 This can also be seen in Fig. 10 which shows that the normalized anti-Stokes signals are essentially independent of the sample gas pressure. This confirms that collisions play no role in the observed increase in Raman signal.

Fluence dependence. The intramolecular vibrational energy distribution after infrared multiphoton excitation depends on the excitation region, which in turn is determined by the infrared pumping intensity. At low fluence the energy is essentially confined to the pumping mode, just as in ordinary one photon spectroscopy (region I). In this case the energy of other modes does not change after excitation, and the Raman signals from these modes simply reflect the thermal population of these modes. At higher fluences the molecule may absorb many infrared photons. In this high excitation region the vibrational modes are strongly coupled, and the nonresonant modes also acquire energy during the excitation (region II). At even higher pumping fluence, dissociation of the molecules occurs (region III). Dissociation fragments, which also contain information on the intramolecular vibrational energy distribution, have been studied by many groups. ${ }^{11,31-33}$ Except for the fluorescence spectrum of infrared multiphoton dissociated $\mathrm{CF}_{2} \mathrm{Cl}_{2}$ all the experiments discussed in this paper were done in region II.

Figure 10 shows the infrared fluence dependence of infrared multiphoton excited $\mathrm{SF}_{6}$ for two pulse durations. Larger signals are obtained for the shorter (higher intensity) pulses, in particular in the low fluence region. At low excitation, a high intensity is needed to overcome the anharmonic shifts, while in the high excitation region, when other nonresonant modes participate in the excitation process, intensity effects become less pronounced. This is consistent with the observation of a 'bottleneck effect' in other experiments. ${ }^{18}$

In contrast to the nearly linear fluence dependence of $\mathrm{SF}_{6}, \mathrm{CF}_{2} \mathrm{Cl}_{2}$ shows an exponential dependence. As shown in Fig. 11, the excitation is a steep function of the pumping fluence. Above $1 \times 10^{4} \mathrm{~J} / \mathrm{m}^{2}$, the signals double roughly every $0.3 \times 10^{4} \mathrm{~J} / \mathrm{m}^{2}$ increment. Since $\mathrm{CF}_{2} \mathrm{Cl}_{2}$ is smaller than $\mathrm{SF}_{6}$, fewer vibrational modes are available and one expects a stronger bottleneck effect in $\mathrm{CF}_{2} \mathrm{Cl}_{2}$. The observed slow rise of the signals at low 
fluence, which is in sharp contrast with the linear fluence dependence of $\mathrm{SF}_{6}$, indeed suggests that this is the case. Measurements of the Raman signal for shorter pulses would provide a better understanding of the role of intensity effects.

Raman intensities. We will now use the normalized anti-Stokes signals as a measure of the average number of quanta in the Raman active modes, and evaluate the distribution of energy in the vibrational modes. Clearly, the $\mathrm{CF}_{2} \mathrm{Cl}_{2}$ results provide the most detailed information, since the anti-Stokes signals of three Raman active modes were measured. In equilibrium the intensities of the normalized signals are given by

$$
I_{\mathrm{rel}}=\frac{1}{e^{h v / k T}-1}
$$

and the mode with lowest frequency will have the largest anti-Stokes signal. The results in Figs. 4, 8, and 11, which are tabulated in Table II, however, show that the signal intensities after infrared multiphoton excitation cannot be described by the above expression. Especially the normalized intensity of the pumped vibrational mode at $923 \mathrm{~cm}^{-1}$ is considerably higher than the corresponding intensities of the other two modes: at all fluences most of the energy remains in the pumped mode. In addition, as is clear from Fig. 11 the rate of increase is different for the three modes. From this figure and the data in Table II, it appears that there is a stronger coupling of the pump mode with the $1098 \mathrm{~cm}^{-1}$ mode than with the less energetic $667 \mathrm{~cm}^{-1}$ mode, notably at the high fluence end. Note also that although the intensities of the anti-Stokes signals increase significantly between 1.5 and $2.4 \times 10^{4} \mathrm{~J} / \mathrm{m}^{2}$, the intensity ratio does not change much. This rules out the possibility that the observed nonequilibrium distribution is a result of averaging a 'hot' equilibrium ensemble and a 'cold' bottlenecked ensemble, since the ratio would change as the fraction of molecules in the hot ensemble becomes larger with increasing fluence. Adding up the energy content of the three modes for $\mathrm{CF}_{2} \mathrm{Cl}_{2}$ from Table II, it appears that a complete randomization of energy only occurs above $10,000 \mathrm{~cm}^{-1}$ of excitation.

One expects collisions to relax the nonequilibrium energy distribution induced by the infrared multiphoton excitation. Even though the decay times of the anti-Stokes signals shown in Fig. 8 are unequal, it is not possible to draw any quantitative conclusions from these data because the signals drop below the noise level before equilibrium is reached. When buffer gas is added, the collision rate increases and the energy distribution should reach equilibrium more quickly. The intensity ratios shown in Fig. 12 indeed tend toward equilibrium with increasing buffer gas pressure. 
Table II Average vibrational energy and relative intensity ratio for three Raman active modes at 667,923 and $1098 \mathrm{~cm}^{-1}$ for $\mathrm{CH}_{2} \mathrm{Cl}_{2}$ after infrared multiphoton excitation. The top line gives the (calculated) room temperature equilibrium values. At a $\mathrm{N}_{2}$ buffer gas pressure of $26 \mathrm{kPa}$ the anti-Stokes signal of the $923 \mathrm{~cm}^{-1}$ mode drops below the noise level.

\begin{tabular}{cccccc}
\hline $\begin{array}{c}F \\
\left(10^{4} \mathrm{~J} / \mathrm{m}^{2}\right)\end{array}$ & $\begin{array}{c}p_{\mathrm{N}_{2}} \\
(\mathrm{kPa})\end{array}$ & $I_{\text {rel ratio }}$ & $\begin{array}{r}E_{667} \\
\left(\mathrm{~cm}^{-1}\right)\end{array}$ & $\begin{array}{c}E_{923} \\
\left(\mathrm{~cm}^{-1}\right)\end{array}$ & $\begin{array}{r}E_{1098} \\
\left(\mathrm{~cm}^{-1}\right)\end{array}$ \\
\hline & & & & & \\
0 & - & $3.4: 1: 0.45$ & 28 & 11 & 6 \\
1.2 & - & $0.21: 1: 0.23$ & 70 & 480 & 130 \\
1.5 & - & $0.15: 1: 0.41$ & 120 & 1140 & 560 \\
1.8 & - & $0.12: 1: 0.48$ & 180 & 2160 & 1240 \\
2.1 & - & $0.10: 1: 0.48$ & 280 & 3800 & 2190 \\
2.4 & - & $0.10: 1: 0.48$ & 440 & 6300 & 3620 \\
2.1 & 13 & $0.20: 1: 1$ & 130 & 920 & 1100 \\
2.1 & 26 & $0.17:-: 0.15$ & 110 & $<450$ & 160 \\
\hline
\end{tabular}

At a buffer gas pressure of $13 \mathrm{kPa}$ the relative intensities of the $923 \mathrm{~cm}^{-1}$ and the $1098 \mathrm{~cm}^{-1}$ mode become nearly identical. At $26 \mathrm{kPa}$, equilibrium is established between the $667 \mathrm{~cm}^{-1}$ and the $1098 \mathrm{~cm}^{-1}$ modes. At this pressure, however, the overall signal has decreased so much because of vibration-translation relaxation that the anti-Stokes signal from the pump mode becomes too small to be detected.

Since $\mathrm{SF}_{6}$ has only one Raman active mode, it is not possible to compare the energy content of different modes as for $\mathrm{CF}_{2} \mathrm{Cl}_{2}$. One can nonetheless obtain qualitative information from a comparison of the antiStokes Raman intensity with the result of photoacoustic experiments, which measure the total energy absorbed per molecule. ${ }^{8}$ The comparison is done as follows. First the energy content of the Raman active mode is determined from the magnitude of the normalized anti-Stokes signals. Next the total energy content of all the modes is determined assuming all vibrational modes of $\mathrm{SF}_{6}$ are in equilibrium. As can be seen in Fig. 15 and Table III, the results thus obtained closely match the values obtained from photoacoustic measurements. In other words, the Raman signal corresponds to what one would expect after equipartitioning the absorbed energy among all 
Table III Relative intensity and vibrational energy of the $775 \mathrm{~cm}^{-1}$ vibrational mode of $\mathrm{SF}_{6}$ after infrared multiphoton excitation with $0.5 \mathrm{~ns}$ (top) and $15 \mathrm{~ns}$ (bottom) pulses. The total vibrational energy of the molecules, calculated assuming intramolecular energy, is compared with the result obtained from photoacoustic measurement $\left(E_{\mathrm{PA}}\right)$.

\begin{tabular}{lcrrr}
\hline $\begin{array}{c}F \\
\left(10^{4} \mathrm{~J} / \mathrm{m}^{2}\right)\end{array}$ & $I_{\text {rel }}$ & $\begin{array}{c}E_{775} \\
\left(\mathrm{~cm}^{-1}\right)\end{array}$ & $\begin{array}{c}E_{\text {total }} \\
\left(\mathrm{cm}^{-1}\right)\end{array}$ & $\begin{array}{c}E_{\mathrm{PA}} \\
\left(\mathrm{cm}^{-1}\right)\end{array}$ \\
\hline & & 28 & 11 & 6 \\
0 & - & & & \\
0.5 & 1.1 & 850 & 13000 & 15000 \\
1.0 & 2.0 & 1600 & 23000 & 23000 \\
2.0 & 3.3 & 2700 & 39000 & 39000 \\
4.0 & 5.0 & 3900 & 57000 & 57000 \\
7.0 & 6.8 & 5300 & 78000 & 78000 \\
0.5 & & & & - \\
1.0 & 0.44 & 340 & 5000 & - \\
2.0 & 0.9 & 700 & 10300 & - \\
4.0 & 1.8 & 1400 & 20600 & - \\
\hline
\end{tabular}

vibrational modes. This suggest that for $\mathrm{SF}_{6}$ the intramolecular vibrational energy distribution after collisionless infrared multiphoton excitation is in equilibrium. For a more detailed discussion the reader is referred to a previously published paper. ${ }^{22}$

The other two molecules studied, $\mathrm{CF}_{2} \mathrm{Cl}_{2}$ and $\mathrm{CH}_{3} \mathrm{CHF}_{2}$, show no or almost no changes in Raman spectrum, even though they absorb a significant amount of energy. This indicates that the excitation energy remains mostly in the modes that were not probed, most likely in the pumped mode, just as for the $\mathrm{CF}_{2} \mathrm{Cl}_{2}$.

Fluorescence. As can be seen in Fig. 13, the laser induced fluorescence from the dissociation fragments of infrared multiphoton dissociated $\mathrm{CF}_{2} \mathrm{Cl}_{2}$ extends far into the blue side of the Raman probe. This indicates that the dissociation fragments carry up to $5000 \mathrm{~cm}^{-1}$ of excitation energy, corresponding to five infrared photons. It should also be noted that the shape of the spectrum does not change with increasing fluence. This implies that an increase in fluence does not change the energy distribution of the dissociation fragments, but only increases the number of dissociated 


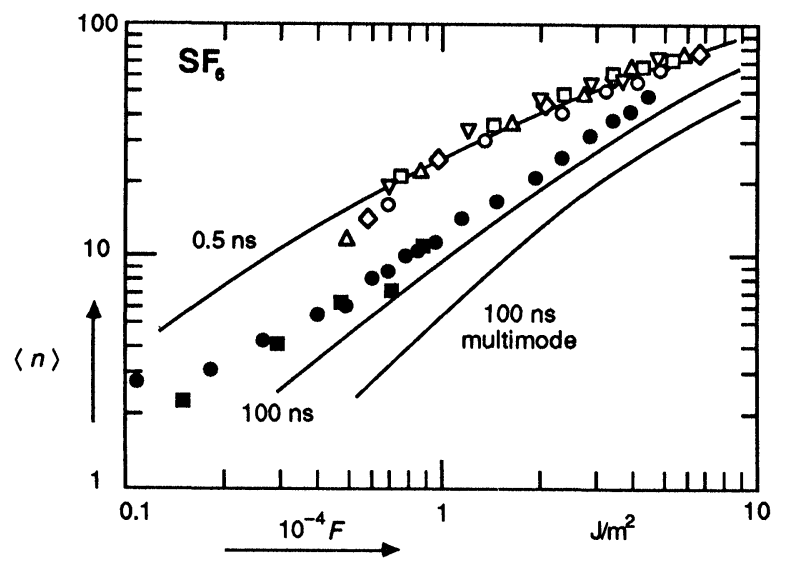

Figure 15 Average number of infrared photons absorbed per molecule as a function of infrared fluence. The data points shown were obtained from the ones shown in Figure 10 , assuming thermal equilibrium between all vibrational modes immediately after the infrared multiphoton excitation. The solid lines are the average number of infrared photons obtained from photoacoustic measurements.

molecules. Therefore the unimolecular dissociation rate of infrared multiphoton excited $\mathrm{CF}_{2} \mathrm{Cl}_{2}$ must be much smaller than the excitation rate.

\section{Conclusion}

This paper presents time-resolved Raman spectra of infrared multiphoton excited molecules. After excitation, collisionless changes in signal intensities are observed for $\mathrm{SF}_{6}, \mathrm{CF}_{2} \mathrm{Cl}_{2}$ and $\mathrm{CH}_{3} \mathrm{CHF}_{2}$. Information on the intramolecular distribution of energy after infrared multiphoton excitation is obtained by using the anti-Stokes Raman intensities as a measure of the energy content of the Raman active vibrational modes. For the molecules studied here, both equilibrium and nonequilibrium energy distributions were observed. Since the signals are averaged over the ensemble of all molecules in the interaction volume, whether highly excited or not, the results presented here are a convolution of the behavior at high excitation with that at low excitation. Still, for $\mathrm{CF}_{2} \mathrm{Cl}_{2}$ the anti-Stokes intensity ratio does not change as the fraction of highly excited molecules is enlarged by increasing the pumping fluence. This leads to the conclusion that even at high excitation the intramolecular vibrational distribution is still 
nonequilibrium, with an excess of energy in the pumped mode. It should be emphasized, however, that these conclusions hold for molecules in region II, and not for dissociating molecules since the signal from those molecules is rejected. Therefore the above conclusions do not contradict the observation ${ }^{11}$ that the infrared multiphoton dissociation of molecules is consistent with RRKM theory. ${ }^{34}$

\section{Acknowledgments}

We are pleased to acknowledge stimulating discussions with Professors N. Bloembergen and M. Quack, and Dr. L. A. Lompré. This work was supported by the Army Research Office and the Joint Services Electronics Program under contracts with Harvard University. ${ }^{35}$

\section{References}

1 V.S. Letokhov, Physics Today 11, 34 (1980)

2 N. Bloembergen and E. Yablonovitch, Physics Today 5, 23 (1978)

3 V.N. Bagratashvili, V.S. Letokhov, A.A. Makarov, E.A. Ryabov, Multiphoton Infrared Laser Photophysics and Photochemistry (Harwood Academic Publishers, New York, 1985)

4 D.S. King, Dynamics of the Excited State, Ed. K.P. Lawley (Wiley, New York, 1982)

5 W. Fuss and K.L. Kompa, Prog. Quant. Electr. 7, 117 (1981)

6 P.A. Schulz, Aa. S Sudbø, D.J. Krajnovitch, H.S. Kwok, Y.R. Shen, and Y.T. Lee, Ann. Rev. Phys. Chem. 30, 379 (1979)

7 C. D. Cantrell, S. M. Freund, J. L. Lyman, Laser Handbook, Vol. 3, Ed. M.L. Stitch (North-Holland, Amsterdam, 1979)

8 J.G. Black, P. Kolodner, M.J. Schultz, E. Yablonovitch, and N. Bloembergen, Phys. Rev. A19, 704 (1979)

9 Y.T. Lee, and Y.R. Shen, Physics Today 33, 52 (1980)

10 J.D. Campbell, G. Hancock, J.B. Halpern, and K.H. Welge, Chem. Phys. Lett. 44, 404 (1976)

11 David S. King and John C. Stephenson, Chem. Phys. Lett. 51, 48 (1977)

12 D.S. Frankel and T.J. Manuccia, Chem. Phys. Lett. 54, 451 (1978)

13 R.C. Sharp, E. Yablonovitch and N. Bloembergen, J. Chem. Phys. 74, 5357 (1981)

14 P. Mukherjee and H.S. Kwok, J. Chem. Phys. 84, 1285 (1986) 
15 V.N. Bagratashvili, Yu.G. Vainer, V.S. Doljikov, S.F. Koliakov, A.A. Makarov, L.P. Malyavkin, E.A. Ryabov, E.G. Silkis, and V.D. Titov, Appl. Phys. 22, 101 (1980)

16 V.N. Bagratashvili, Yu.G. Vainer, V.S. Dolzhikov, S.F. Kol'yakov, V.S. Letokhov, A.A. Makarov, L.P. Malyavkin, E.A. Ryabov, E.G. Sil'kis, and V.D. Titov, Sov. Phys. JETP 53, 512 (1981)

17 V.N. Bagratashvili, V.S. Doljikov, V.S. Letokhov, A.A. Makarov, L.P. Maljavkin, E.A. Ryabov, E.G. Silkis, and Yu.G. Vainer, Opt. Comm. 38, 31 (1981)

18 V.N. Bagratashvili, Yu.G. Vainer, V.S. Doljikov, V.S. Letokhov, A.A. Makarov, L.P. Malyavkin, E.A. Ryabov, and E.G. Sil'kis, Opt. Lett. 6, 148 (1981)

19 Yu.S. Doljikov, V.S. Letokhov, A.A. Makarov, A.L. Malinovsky and E.A. Ryabov, Chem. Phys. Lett. 124, 304 (1986)

20 V.S. Doljikov, Yu.S. Doljikov, V.S. Letokhov, A.A. Makarov, A.L. Malinovsky and E.A. Ryabov, Chem. Phys. 102, 155 (1986)

21 E. Mazur, I. Burak, and N. Bloembergen, Chem. Phys. Lett. 105, 258 (1984)

22 Jyhpyng Wang, Kuei-Hsien Chen, and Eric Mazur, Phys. Rev. A 34, 3892 (1986)

23 Eric Mazur, Rev. Sci. Instrum. 57, 2507 (1986)

24 T.B. Simpson, J.G. Black, I. Burak, E. Yablonovitch and N. Bloembergen, J. Chem. Phys. 83, 628 (1985)

25 G. Herzberg, Molecular spectra and molecular structure, Vol. 2 (Van Nostrand Reinhold, New York, 1979)

26 Charles A. Bradley, Jr., Phys. Rev. 40, 908 (1932)

27 T. Shimanouchi, J. Phys. Chem. Ref. Data 6, 993 (1977)

28 D.W. Lupo, and M. Quack, Chem. Rev. 87, 181 (1987)

29 F. Albert Cotton, Chemical Applications of Group Theory (Wiley-Interscience, New York, 1971)

30 R.D. Bates Jr., J.T. Knudtson, G.W. Flynn and A.M. Ronn, Chem. Phys. Lett. 8, 103 (1971)

31 J.W. Hudgens, J. Chem. Phys. 68, 777 (1978)

32 R.J.S. Morrison and E.R. Grant, J. Chem. Phys. 71, 3573 (1979)

33 Aa.S. Sudbø, P.A. Schulz, E.R. Grant, Y.R. Shen, and Y.T. Lee, J. Chem. Phys. 70, 912 (1979)

34 Benson S.W. Chem. Rev. 78 23(1978)

35 Contract numbers: DAAG29-85-K-0600 and N00014-84-K-0465. 